New windows on massive stars: asteroseismology, interferometry, and spectropolarimetry

Proceedings IAU Symposium No. 307, 2014

G. Meynet, C. Georgy, J. H. Groh 8 Ph. Stee, eds.

(C) International Astronomical Union 2015 doi:10.1017/S1743921314007200

\title{
Spectropolarimetry and modeling of WR156
}

\author{
Olga Maryeva \\ Special Astrophysical Observatory of the Russian Academy of Sciences \\ email: olga.maryeva@gmail.com
}

\begin{abstract}
For the first time spectropolarimetric observations of Wolf-Rayet star WR156 (WN8h) were conducted. Medium resolution spectropolarimetric data in the range of 3500-7200 Å were obtained at Russian 6-m telescope of Special Astrophysical Observatory (SAO RAS). These data show that the light from the star is significantly polarized, with the degree of polarization $P=1.38 \pm 0.06 \%$, and the position angle $\Theta=77.4^{\circ} \pm 1.2^{\circ}$. This polarization, most probably, has an interstellar origin, as its magnitude and orientation are similar to the ones of field stars. Also, we present results of numerical modeling of WR156 atmosphere performed using CMFGEN code. According to it, WR156 is the richest hydrogen Wolf-Rayet star of WN8 type in the Galaxy.
\end{abstract}

Keywords. stars : individual : WR156; stars : Wolf-Rayet; techniques : polarimetric

\section{Introduction}

Even for spatially unresolvable objects, linear spectropolarimetry with moderate resolution is a powerful tool to detect and investigate the asymmetric structure of the object and to search rapid rotation. The linear polarization (greater than $0.3 \%$ ) was found in 29 WR stars (i.e. almost in 15\% of Galactic WR stars, see Harries et al. 1998), but the "line effect" is identified only in six WR stars (Harries et al. 1998). Moreover, some WR stars demonstrate polarimetric continuum variability due to small-scale structure of the wind. Combining the results of spectropolarimetric observations with numerical simulations of stellar winds may be the best way for understanding the cause of the appearance of the linear polarization in WR stars, and to reveal the mechanisms of the "line effect".

\section{Study of the Galactic Wolf-Rayet star WR 156 (WN8h)}

Spectropolarimetry of of WR156 was performed at the Russian 6-m telescope. Measured degree of polarization is $P=1.38 \pm 0.06 \%$, position angle is $\Theta=77.4 \pm 1.2^{\circ}$. The spectrum does not show any signs of reduction in polarization at the wavelengths corresponding to emission lines, i.e. "lines effects" are not detected. Estimation of interstellar polarization (ISP) is very important for the study of stellar polarizations. Using the field stars we created a map of the interstellar polarization and we concluded, that, most likely, the polarization measured by us has an interstellar origin.

Using CMFGEN code we have constructed two models of the WR156 atmosphere for different values of the bolometric luminosity of $L_{\star}$. In the calculation of the first model, we assumed that the $L_{\star}=5.3 \cdot 10^{5} L_{\odot}$ of WR156 is equal to the one of FSZ35, which is located in the galaxy M33 and is also classified as WN8h. The second model was constructed for $L_{\star}=3.3 \cdot 10^{5} L_{\odot}$. Table 1 lists the parameters of both models. In the optical range both models show almost identical spectra. Also the table shows parameters of other WN8h whose are derived using CMFGEN. Luminosity and mass-loss rate of WR156 are similar to the ones of WR40 and WR16 stars, displaying the line effect, but the temperature and the abundance of hydrogen are significantly different from them. Apparently, present 


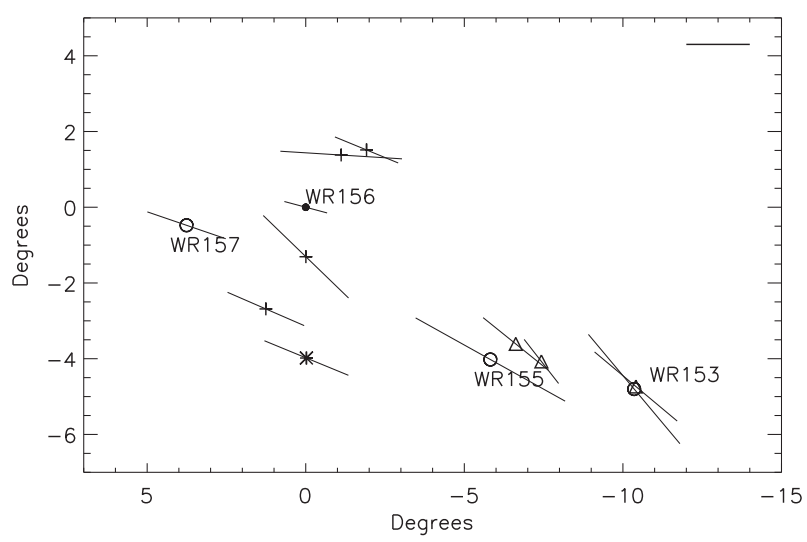

Figure 1. The interstellar polarization map around WR156.

Table 1. Parameters of atmospheres of WR156 and other WN8h stars in the Milky Way and M33 galaxy (FSZ35 and Romano's star, in minimum of brightness). $X_{\mathrm{H}}, X_{\mathrm{He}}, X_{\mathrm{C}}$ and $X_{\mathrm{N}}$ are the mass fractions of hydrogen, helium, carbon and nitrogen, respectively.

\begin{tabular}{lcccccccccc}
\hline & $\begin{array}{c}T_{\star} \\
{[\mathrm{kK}]}\end{array}$ & $\begin{array}{c}R_{\star} \\
{\left[R_{\odot}\right]}\end{array}$ & $\begin{array}{c}\log L_{\star} \\
{\left[L_{\odot}\right]}\end{array}$ & $\begin{array}{c}\log \dot{M}_{\mathrm{cl}} \\
{\left[M_{\odot} \mathrm{yr}^{-1}\right]}\end{array}$ & $\begin{array}{c}v_{\infty} \\
{\left[\mathrm{km} \mathrm{s}^{-1}\right]}\end{array}$ & $\begin{array}{c}X_{\mathrm{H}} \\
{[\%]}\end{array}$ & $\begin{array}{c}X_{\mathrm{He}} \\
{[\%]}\end{array}$ & $\begin{array}{c}X_{\mathrm{C}} \\
{[\%]}\end{array}$ & $\begin{array}{c}X_{\mathrm{N}} \\
{[\%]}\end{array}$ & Ref \\
\hline WR124 & 32.7 & 18.0 & 5.53 & -4.7 & 710 & 15 & & & & {$[1]$} \\
WR40 & 45.0 & 10.6 & 5.61 & -4.5 & 840 & 15 & 83 & 1.2 & 1.12 & {$[2]$} \\
WR16 & 41.7 & 12.3 & 5.68 & -4.8 & 650 & 23 & 75 & & & {$[2]$} \\
FSZ35 & 36.5 & 19 & 5.76 & -4.58 & 800 & 16.5 & 82 & 0.75 & 0.3 & {$[3]$} \\
Romano's & 34.0 & 20.8 & 5.7 & -4.72 & 360 & 31.8 & 67 & 2 & 0.7 & {$[4]$} \\
star & & & & & & & & & & \\
WR156 & 36 & 18.7 & 5.72 & -4.82 & 650 & 33 & 65.8 & 6 & 0.7 & {$[5]$} \\
WR156 & 36 & 14.8 & 5.52 & -5 & 650 & 33 & 65.8 & 6 & 0.7 & {$[5]$} \\
\hline
\end{tabular}

Notes: [1]- Crowther et al. (1999), [2]- Herald et al. (2001), [3]- Maryeva \& Abolmasov (2012b), [4]- Maryeva \& Abolmasov (2012a), [5]- Maryeva et al. (2013)

statistics on WR stars with line effects is so small that it does not allow to define any relation between the line effect and some parameters of the stellar atmosphere.

Results are published in Maryeva et al. (2013).

\section{Acknowledgements}

Olga Maryeva thanks the International Astronomical Union and Dynasty Foundation for grants. The study was supported by the Russian Foundation for Basic Research (projects no. 14-02-31247,14-02-00291).

\section{References}

Crowther, P. A., Pasquali, A., De Marco, O., et al. 1999, A $\& A$ 350, 1007

Harries, T. J., Hillier, D. J., \& Howarth, I. D. 1998, MNRAS 296, 1072

Herald, J. E., Hillier, D. J., \& Schulte-Ladbeck, R. E. 2001, ApJ 548, 932

Maryeva, O. \& Abolmasov, P. 2012a, MNRAS 419, 1455

Maryeva, O. \& Abolmasov, P. 2012b, MNRAS 421, 1189

Maryeva, O. V., Afanasiev, V. L., \& Panchuk, V. E. 2013, New. Astron. 25, 27 